\title{
BROTE NOSOCOMIAL DE QUERATOCONJUNTIVITIS EPIDÉMICA EN UNA UNIDAD DE CUIDADOS INTENSIVOS NEONATAL
}

\author{
NOSOCOMIAL OUTBREAK OF EPIDEMIC \\ KERATOCONJUNCTIVITIS IN A NEONATAL INTENSIVE CARE \\ UNIT
}

\author{
ASENCIO-DURÁN M ${ }^{1}$, ROMERO-MARTÍN R ${ }^{1}$, GARCÍA-MARTÍNEZ JR ${ }^{1}$, PERALTA-CALVO JM ${ }^{2}$, \\ PÉREZ-BLANCO V ${ }^{3}$, GARCÍA-CABALLERO J ${ }^{3}$, MALO-MARTÍNEZ C ${ }^{4}$
}

\begin{abstract}
RESUMEN
Objetivos: Descripción de un brote nosocomial epidémico de queratoconjuntivitis ocurrido en Madrid (Unidad de Cuidados Intensivos Neonatal) entre los meses de mayo y octubre de 2002 y que afectó al menos a 43 trabajadores del centro; investigación del posible origen y valoración de secuelas. Medidas preventivas para evitar nuevos brotes.

Material y métodos: Estudio descriptivo del brote, definición de caso clínico, investigación microbiológica y epidemiológica mediante encuesta. Análisis de la relación entre la tasa de infiltrados subepiteliales 1 año después de la infección y la tasa de uso de corticoide tópico.

Resultados: El brote epidémico empezó en el Servicio de Neonatología, encontrándose como focos iniciales unos gemelos prematuros ingresados en dicha unidad. La diseminación se produjo en 24 trabajadores de la unidad, y otros 19 trabajadores de otros Servicios. El diagnóstico microbiológico con-
\end{abstract}

\begin{abstract}
Objective: To describe an epidemic nosocomial outbreak of keratoconjunctivitis affecting at least 43 people in a Neonatal Intensive Care Unit in Madrid, between May and October 2002, perform epidemiologic research and determine preventive measures to prevent new outbreaks.

Methods: Description of the outbreak, clinical case definition, microbiologic and epidemiologic research through the use of questionnaires. Analysis of the incidence of subepithelial infiltrates 1 year after infection and the use of topical corticosteroid therapy.

Results: The epidemic began in the Neonatal Service, with a pair of twins being found as the initial focus. Dissemination occurred to about 24 workers of the unit, and 19 workers from other services. The microbiologic diagnosis confirmed adenovirus in 10 conjunctival smears and one serologically, with the remainder obeying clinical and epidemiological criteria of such infection. Infected patients using
\end{abstract}

\footnotetext{
Recibido: 2/2/04. Aceptado: 18/1/07.

Hospital General Universitario La Paz. Madrid. España.

1 Licenciado en Medicina. Servicio de Oftalmología.

2 Doctor en Cirugía. Servicio de Oftalmología Infantil.

3 Doctor en Medicina. Servicio Medicina Preventiva.

4 Licenciado en Medicina. Servicio Salud Laboral.

Comunicación presentada en el LXXIX Congreso de la S.E.O. (Valencia 2003).

Correspondencia:

Mónica Asencio Durán

C/. Francos Rodríguez, 84, 2. ${ }^{\circ} \mathrm{C}$

28039 Madrid

España

E-mail: masedur@terra.es
} 
firma infección por adenovirus en 10 exudados conjuntivales y una serología, el resto cumple criterios clínicos y epidemiológicos. Aunque es una muestra pequeña y no concluyente, en este estudio los pacientes que usaron corticoide tópico tienen mayor tasa de infiltrados que los que no usaron $(\mathrm{p}=0,003$ en test exacto de Fisher).

Conclusiones: La queratoconjuntivitis epidémica es una enfermedad frecuente y en la mayoría banal, pero su alta tasa de contagiosidad y sus posibles secuelas requieren que se preste mayor atención a las medidas preventivas para evitar nuevos brotes.

Palabras clave: Queratoconjuntivitis epidémica, brote nosocomial, Adenovirus, infiltrados subepiteliales. topical corticosteroids had a higher incidence of infiltrates 12 months later than those not using such agents ( $p=0.003$ in Fisher test).

Conclusions: Epidemic keratoconjunctivitis occurs frequently, is highly contagious and has possible long-term sequelae, necessitating the use of preventive measures to avoid spread and new outbreaks (Arch Soc Esp Oftalmol 2007; 82: 73-80).

Key words: Epidemic keratoconjunctivitis, nosocomial outbreak, Adenovirus, subepithelial infiltrates.

\section{INTRODUCCIÓN}

La queratoconjuntivitis epidémica (QCE) es una patología frecuente causada por Adenovirus, virus ADN bicatenario sin envoltura, muy resistente a agentes externos. De inicio súbito, se manifiesta inicialmente como una conjuntivitis aguda con lagrimeo, ojo rojo, malestar y fotofobia, bilateral en el $60 \%$ (1). A la exploración se objetiva edema palpebral con secreción acuosa, quémosis y reacción folicular conjuntival, en casos graves también hemorragias subconjuntivales, pseudomembranas o linfadenopatía dolorosa. El tratamiento es sintomático ya que la enfermedad cura espontáneamente en dos semanas. La queratitis aparece más tarde, y se manifiesta como sensación de arenilla, disminución de agudeza visual, fotofobia, lagrimeo o blefarospasmo, y a partir de los 10 días de ésta aparecen los típicos infiltrados subepiteliales entre el 35\%-38\%, constituyendo una de las complicaciones más graves de la QCE, ya que pueden disminuir la agudeza visual; sin tratamiento suelen desaparecer, aunque pueden persistir de meses a años $(2,3)$.

La QCE se transmite a través de secreciones oculares de pacientes afectos, siendo reservorio el hombre. Tras un período de incubación de 4 a 10 días se considera que lágrima y saliva son contagiosas durante un periodo de unas 2 semanas. Se describen dos síndromes asociados:

- Fiebre faringoamigdalar (FFA): causada por los Adenovirus 3, 7 y 5, produce infección de vía aérea superior en niños; y en menor proporción, queratitis leve.
- Queratoconjuntivitis epidémica (QCE): causada por los serotipos 8,19 y 10 . Produce queratitis en el $80 \%$, que puede ser grave.

El diagnóstico diferencial debe establecerse con las conjuntivitis hemorrágicas por enterovirus y la conjuntivitis por chlamydia. No existe tratamiento específico para la QCE, aunque se han ensayado tratamientos como cidofovir tópico al $0,2 \%$ y ciclosporina al $1 \%$, sin resultados concluyentes $(4,5)$.

Los niños prematuros y los pacientes inmunocomprometidos pueden ser portadores del virus durante largos períodos de tiempo y ser causa de infección en otros pacientes de alto riesgo. La presentación de la QCE puede ser esporádica o en brotes (6-8), sobre todo en comunidades cerradas, de distribución mundial y en cualquier época del año. Existen en la literatura varios casos de brotes nosocomiales, la mayoría en unidades de críticos o en departamentos pediátricos (9), siendo una fuente de infección frecuente una consulta de oftalmología (1,10-14), por transmisión directa a través de secreciones oculares infectadas, o indirecta, a través de fómites, como las manos $(1,12)$ del personal sanitario, o los instrumentos de exploración oftalmológica como la lámpara de hendidura, el tonómetro o las lentes de contacto (15).

El objetivo del estudio es la descripción clínica y epidemiológica del brote de QCE ocurrido en la UCI Neonatos de nuestro centro entre los meses de mayo y octubre de 2002, descripción del posible origen infeccioso, valoración de secuelas un año después y su relación con el tra- 
tamiento. Descripción de las medidas preventivas instauradas para la prevención de otros brotes nosocomiales.

\section{SUJETOS, MATERIAL Y MÉTODO}

Una vez identificado el brote se realizó un estudio epidemiológico descriptivo para la caracterización en tiempo, espacio y persona. Se obtuvieron los listados de personal afectado de las historias recogidas en Urgencias, Consultas Externas de Oftalmología y Servicio de Salud Laboral. Se definieron como casos:

Caso confirmado: diagnosticado mediante pruebas de laboratorio concluyentes (serología o exudado conjuntival positivo en al menos un ojo). Caso probable: clínica compatible de QCE durante el período en el que se presentó el brote, siendo población a riesgo y trabajando en el hospital. Caso primario: caso confirmado, probable o sospechoso caracterizado por haber comenzado los síntomas durante un período de tiempo compatible con incubación a partir del contacto con la considerada fuente de infección. Caso secundario: aquél relacionado con un caso primario posterior a un tiempo compatible con el de incubación.

Se diseñó una hoja de recogida de datos con varios apartados: variables socio-demográficas, variables clínicas, pruebas diagnósticas, tratamiento realizado, variables epidemiológicas y valoración de secuelas. En el apartado de las variables epidemiológicas se recogieron datos sobre el lugar de trabajo, mecanismos de transmisión y entorno del paciente. El cuestionario se contestó tras entrevista personal y exploración realizada por oftalmólogos previa llamada telefónica, entre los meses de noviembre de 2002 a marzo de 2003. Posteriormente se realizó una base de datos en el programa Access 97 (Microsoft Access for Windows, Chicago, USA) y exportación al paquete estadístico SPSS.10 (SPSS for Windows, SPSS Inc., Chicago, USA) para análisis descriptivo de todas las variables; las cuantitativas se describen como media y las cualitativas como número de casos y porcentajes.

Se recogieron frotis conjuntivales para el aislamiento de Adenovirus y sangre para serología (ELISA frente a Adenovirus, CMV, VHS y VVZ), solamente los casos en que los pacientes acudieron primero al Servicio de Medicina Laboral.

\section{RESULTADOS}

\section{Descripción epidemiológica y sociodemográfica}

El número de pacientes estudiados fue 43 . El número de casos propiamente del brote fueron 40 , el primero diagnosticado el 5 de mayo de 2002 y el último el 20 de agosto de 2002, ya que tres se pueden considerar aislados al ser diagnosticados en marzo y en octubre (fig. 1). La curva epidémica es bimodal, con dos picos en la $4 .^{\mathrm{a}}$ semana de mayo y la $2 .^{a}$ de junio, esto puede deberse a que el segundo pico sea de casos secundarios, al encontrarse entre los días del periodo de incubación (4 a 10 días). La distribución según profesión (tabla I) y según el lugar de trabajo (tabla II). La distribución por sexo fue de cinco $(11,6 \%)$ varones y $35(88,4 \%)$ mujeres, la edad media de 41,2 años y la mediana de 40 años. El tiempo medio estimado de inicio de los síntomas desde el probable contacto fue de 5,65 días. En casos bilaterales, el tiempo medio de evolución entre uno y otro ojo fue de 5,74 días.

Recogida de muestras: En 16 casos se recogieron frotis conjuntivales (cinco bilaterales y 11 unilaterales; de los bilaterales fueron positivos para ambos ojos dos, negativos para ambos ojos dos, y un ojo positivo: uno; de los 11 unilaterales, seis fueron

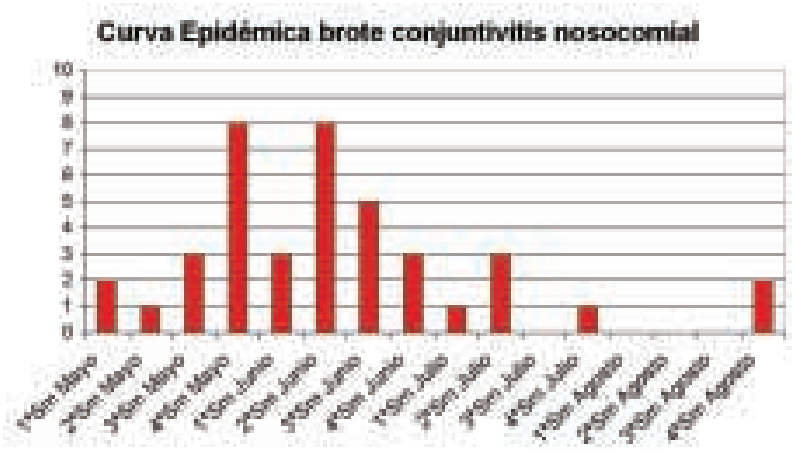

Fig. 1: Curva del brote.

Tabla I. Distribución según profesión

\begin{tabular}{lcc}
\hline Profesión & Frecuencias & Porcentajes \\
\hline Médico & 9 & 20,9 \\
DUE & 17 & 39,5 \\
Auxiliar clínica & 9 & 20,9 \\
Otros & 8 & 18,6 \\
Total & 43 & 100,0 \\
\hline \hline
\end{tabular}


Tabla II. Distribución según lugar de trabajo

\begin{tabular}{lcc}
\hline Lugar de trabajo habitual & Frecuencias & Porcentajes \\
\hline UCI neonatal & 8 & 18,6 \\
Vigilancia Intensiva Neonatal & 3 & 7,0 \\
Planta neonatos & 13 & 30,2 \\
Otro & 19 & 44,2 \\
Total & 43 & 100,0 \\
\hline \hline
\end{tabular}

positivos y cinco negativos). En 16 pacientes se recogió serología (15 negativas y una positiva).

Confirmación y descripción del brote: 30 casos probables (cumplen criterios clínicos y epidemiológicos) y 10 casos confirmados (una serología positiva y nueve exudado conjuntival positivo en al menos un ojo).

Un dato a considerar es que el $88 \%$ de los encuestados cree conocer el lugar de contagio: en un $58 \%$ el propio Servicio de Neonatología y en $31,2 \%$ en otros lugares (sólo tres casos o $7 \%$ en consultas de Oftalmología). Afirmaron tener contacto previo con trabajadores del brote el $58,1 \%$, y con afectados fuera del ambiente hospitalario el $14 \%$, siendo llamativo el hecho de que los 15 primeros trabajadores (todos del Servicio de Neonatología) recuerdan aproximadamente la cadena de transmisión del virus. El tipo de contacto probable fue en la mayoría de casos indirecto $(51,2 \%)$, y directo en apenas ocho casos $(18,6 \%)$.

Investigación epidemiológica. Tras entrevistar a los trabajadores afectados se llegó a la conclusión de que el brote pudo empezar en la UCI Neonatal a partir de dos gemelos prematuros con retinopatía de la prematuridad. No se llegó a determinar cuál de ellos fue el primer afectado, pero se cree que la transmisión de uno a otro se produjo a través del blefarostato con el que se exploró a estos pacientes y una eventual mala esterilización del mismo. Se documentan otros dos casos de niños críticos nacidos a término y no explorados por un oftalmólogo que también la padecieron y que junto con los prematuros se han etiquetado de focos de infección primarios. Posteriormente aparecen 15 casos primarios en el personal sanitario de dicha unidad y otras unidades del Servicio de Neonatología (VIN y Planta). Se han documentado 16 casos secundarios, 11 de los cuales dentro del propio Servicio de Neonatología. El resto de casos no se ubican en estas categorías y pueden corresponder a una tercera onda o a casos aislados.

\section{Descripción clínica y secuelas}

La mayoría de los informes se extrajeron de Urgencias (86\%). El 70\% de los casos fueron bilaterales (30) y los síntomas y signos más frecuentes en la primera visita son detallados en la tabla III. Se emplearon colirios de antibiótico en todos (cloranfenicol, rifamicina u otro), más AINE tópico en $34,9 \%$, Corticoide tópico en 32,6\% y lágrimas artificiales en el 27,9\%; algunos pacientes requirieron varios tratamientos.

En cuanto a las secuelas en estos pacientes 1 año después de la infección, los síntomas y signos más frecuentes se detallan en la tabla IV, siendo la más valorada la sequedad, y en general de carácter leve. El tiempo de duración de las mismas: 1 a 7 días en $16 \%$, más de 1 semana 19\%, más de 4 semanas $32 \%$ y mantenidas al año $11 \%$. Requirieron tratamiento el $55,8 \%$ de los pacientes, siendo el más habitual la lágrima artificial en 22 casos $(51,2 \%)$, corticoide tópico en $11,6 \%$ y suero fisiológico $9 \%$. El porcentaje de pacientes que requirieron uso de lágrima artificial al año de evolución aumentó en más del $20 \%$ con respecto al inicio. Al cruzar los datos de los pacientes que usaron corticoide tópico en las primeras fases de la enfermedad frente a los que no usaron y la tasa de aparición de infiltrados

Tabla III. Síntomas y signos iniciales

\begin{tabular}{lcc}
\hline Síntoma inicial & Frecuencias & Porcentajes \\
\hline Legañas & 30 & $70,8 \%$ \\
Sens. cuerpo extraño & 20 & $47,2 \%$ \\
Secreción & 19 & $44,2 \%$ \\
Lagrimeo & 18 & $41,9 \%$ \\
Edema palpebral & 16 & $37,2 \%$ \\
Dolor & 14 & $33,3 \%$ \\
Picor & 12 & $28,0 \%$ \\
Adenopatías dolorosas & 12 & $27,9 \%$ \\
$\downarrow$ Agudeza visual & 13 & $30,7 \%$ \\
Sintomatología catarral & 10 & $23,0 \%$ \\
Irritación & 8 & $19,4 \%$ \\
Pinchazos & 2 & $5,1 \%$ \\
Mala adaptación a LC & 2 & $5,1 \%$ \\
Cefalea & 2 & $5,1 \%$ \\
Malestar general & 2 & $5,1 \%$ \\
Ojo rojo & 36 & $83,7 \%$ \\
Secreción & 19 & $44,2 \%$ \\
Folículos conjuntivales & 18 & $41,9 \%$ \\
Microhemorragias & 11 & $25,6 \%$ \\
Queratitis & 8 & $18,6 \%$ \\
Quémosis & 7 & $16,3 \%$ \\
Infiltrados subepiteliales & 3 & $7 \%$ \\
Pseudomembranas & 1 & $2,3 \%$ \\
Hiposfagma & 0 & $0 \%$ \\
\hline \hline
\end{tabular}


Tabla IV. Síntomas y signos al año

\begin{tabular}{lrc}
\hline Síntomas & Frecuencias & Porcentajes \\
\hline Sensación de sequedad & 22 & $51,2 \%$ \\
Sensación de CE & 10 & $23,3 \%$ \\
Erosiones recurrentes & 9 & $21 \%$ \\
Hiperemia & 7 & $16,3 \%$ \\
Mala adaptación a LC & 3 & $7 \%$ \\
Picor & 3 & $7 \%$ \\
Lagrimeo & 2 & $4,7 \%$ \\
Fotofobia & 2 & $4,7 \%$ \\
Disminución del BUT & 17 & $39,5 \%$ \\
Infiltrados subepiteliales & 5 & $11,6 \%$ \\
Queratitis punctata & 4 & $9,3 \%$ \\
Ptosis palpebral & 1 & $2,3 \%$ \\
Reacción papilar conjuntival & 1 & $2,3 \%$ \\
Blefaritis & 1 & $2,3 \%$ \\
Cicatrices estrelladas conj & 1 & 2,3 \\
\hline \hline
\end{tabular}

corneales posterior, se halló que los que usaron CE el $50 \%$ tiene infiltrados 1 año después, frente a el $6,9 \%$ de los que no usaron con una diferencia estadísticamente significativa en el test exacto de Fisher ( $\mathrm{p}$ de 0,003 ).

\section{Medidas de control}

Las empleadas habitualmente en estos casos, como:

- Asepsia rigurosa en las Consultas de Oftalmología del Hospital, no permitiéndose la entrada a todas aquellas personas sospechosas de QCE.

- Asistencia de los casos sospechosos en un box separado de Urgencias.

- Recomendación de uso de guantes desechables y lavado riguroso de manos del personal sanitario, desecho de colirios utilizados en contacto con secreciones oculares, así como evitación de exploraciones innecesarias en estos pacientes (15).

- Desinfección de dispositivos médicos, superficies y mobiliario que hubieran podido estar en contacto con secreciones conjuntivales y nasales.

- Baja laboral por el Servicio de Salud Laboral del Hospital, de 15 días desde el inicio de la sintomatología para todos los afectados, incluido el personal de Oftalmología afectado.

\section{DISCUSIÓN}

La QCE es una enfermedad frecuente y en muchos casos banal, por lo que existen pocos estu- dios, no obstante su alta tasa de contagio posee a veces efectos devastadores, como es el caso de las epidemias en comunidades hospitalarias, afectando a personal sanitario que no puede tener contacto con pacientes para evitar la diseminación (16), con el problema laboral que ello conlleva. En este brote nosocomial destacan varios aspectos:

1) Brote producido fuera de un servicio de oftalmología: sólo un $7 \%$ recuerda una visita previa al oftalmólogo. Existen otros brotes documentados en UCI pediatricas, pero con una repercusión menor (9).

2) Alta y rápida contagiosidad a pesar de las estrictas medidas de higiene de esta Unidad. La mayoría de los afectados recuerda la cadena de transmisión del virus en sus comienzos.

Se trata de un virus muy estable sobre superficies inanimadas durante períodos prolongados de tiempo, incluso 49 días en superficies de plástico y metal y hasta 10 días en ropa o papel (17). No es inactivado por detergentes pero sí por soluciones alcohólicas al $70 \%$ o de Clorhexidina, que sería lo recomendable; no obstante, estudios demuestran que el autoclave es el método ideal para la esterilización de blefarostatos en el examen de fondo de ojo en prematuros, quedando como alternativa la desinfección con gluconato de clorhexidina o isopropil alcohol al 70\% $(18,19)$. Además, se deben extremar las medidas de higiene en unidades de riesgo para evitar la diseminación.

En ocasiones las secuelas que produce son molestas y duraderas, afectando a la calidad de vida de los pacientes, que requieren de visitas frecuentes al oftalmólogo, diversos tratamientos, y a veces, continuar de baja laboral. Un año después, destaca el aumento en el uso de lágrimas artificiales en estos pacientes, que se explica por el ojo seco evaporativo al alterarse la secreción de las glándulas conjuntivales. Al contrario que la literatura vigente $(3,20,21)$, se observa una aumento de la tasa de infiltrados en pacientes que usaron corticoide tópico sin AINE desde el inicio, pero hay que decir que se trata de una muestra pequeña (11 casos) y que el tipo de estudio no es adecuado (requeriría un estudio prospectivo de casos y controles).

Una limitación relativa a este estudio es que el diagnóstico no está confirmado en todos los pacientes; no hay estudio microbiológico en todos ellos, ni se complementó con estudio de bacterias u otros virus o se serotipó el Adenovirus; tampoco hay cultivos de fómites como superficies, colirios, instru- 
mentos de exploración oftalmológica ni de manos de pacientes o sanitarios.

A partir de este brote de gran repercusión laboral y mediática se inició una investigación epidemiológica con notificación precoz de casos de QCE y una campaña informativa de prevención en colaboración con los Servicios de Medicina Preventiva y Salud Laboral del Centro, en forma de carteles que se distribuyeron por zonas de riesgo del Hospital (fig. 2).

\section{AGRADECIMIENTOS}

A las personas que han colaborado.

\section{BIBLIOGRAFÍA}

1. Arffa RC. Grayson. Enfermedades de la córnea. IV ed. St Louis: Mosby; 1999.

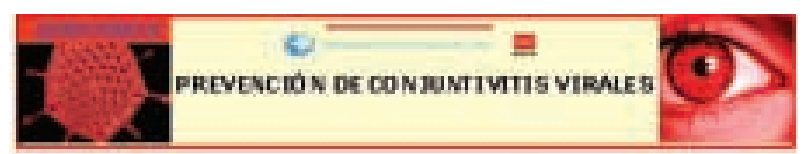

Los Adanovius pueden pamainecer en las superf cies imasas. telblonos, capdat durante varios das, socre la ropa uncs 10 das y en be instumertos diarroiggcos

Las menos del personel santerio comaminedes con secieciones procedartes do un pociente fectado, son in reinciost tuerte do transmision
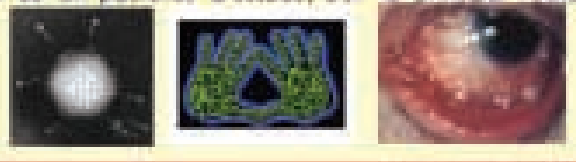

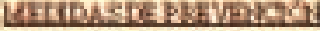

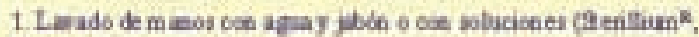

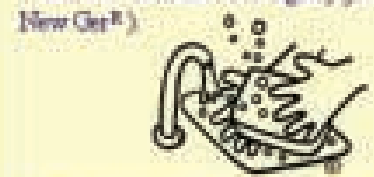

2. Cmbio \& puartet atrepatientry paciente

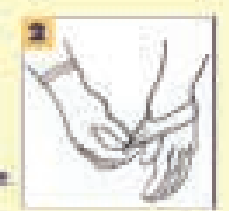

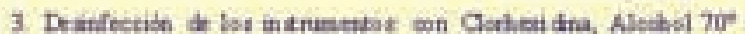

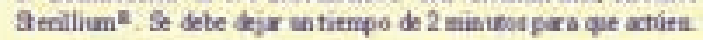
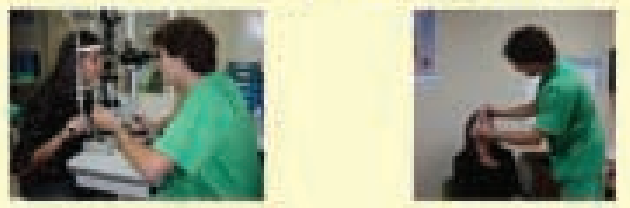

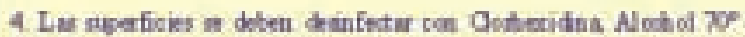
Sterilium"y ojar 2 mimuberpara que actóen. ie ictoen.

Fig. 2: Carteles.
2. Sundmacher R, Engelskirchen U. Recurrent and persistent nummuli after epidemic keratoconjunctivitis. Klin Monatsbl Augenheillkd 1991; 198: 550-554.

3. Hodge W, Wohl T, Whitcher JP, Margolis TP. Corneal subepithelial infiltrate recurrence sine adenovirus. Cornea 1995; 14: 324-325

4. Hillenkamp J, Reinhard T, Ross RS, Bohringer D, Cartsburg $O$, Roggendorf $M$, et al. Topical treatment of acute adenoviral keratoconjunctivitis with $0.2 \%$ cidofovir and 1\% cyclosporine: a controlled clinical pilot study. Arch Ophthalmol 2001; 119: 1487-1491.

5. Hillenkamp J, Reinhard T, Ross RS, Bohringer D, Cartsburg $O$, Roggendorf $M$, et al. The effects of cidofovir $1 \%$ with and without cyclosporin a $1 \%$ as a topical treatment of acute adenoviral keratoconjunctivitis: a controlled clinical pilot study. Ophthalmology 2002; 109: 845-850.

6. Bolyard EA, Tablan OC, Williams WW, Pearson ML, Shapiro CN, Deitchmann SD. Guideline for infection control healthcare personnel, 1998. Hospital Infection Control Practices Advisory Committee. Infect Control Hosp Epidemiol 1998; 19: 407-463.

7. Salcedo MA, Goldaracena B, Ardanaz ME, Mazon A, Moreno $C$, Salvo $S$. Brote nosocomial y comunitario de queratoconjuntivitis epidémica en Navarra en el año 1996. Rev Esp Salud Publica 1997; 71: 383-390.

8. Garcia J, Astier P, Polo ME, Escobar E. Estudio de brotes nosocomiales. Anales del sistema sanitario de Navarra 2000; 23: 49-68.

9. Birenbaum E, Linder N, Varsano N, Azar R, Kuint J, Spie$\operatorname{rer} A$, et al. Adenovirus type 8 conjunctivitis outbreak in a neonatal intensive care unit. Arch Dis Child 1993; 68: 610-611.

10. Jernigan JA, Lowry BS, Hayden FG, Kyger SA, Conway $B P$, Groschel $M$, et al. Adenovirus type 8 epidemic keratoconjunctivitis in an eye clinic: risk factors and control. J Infect Dis 1993; 167: 1307-1313.

11. Warren D, Nelson KE, Farrar JA, Hurwitz E, Hierholzer $J$, Ford E, Anderson LJ. A large outbreak of epidemic keratoconjunctivitis: problems in controlling nosocomial spread. J Infect Dis 1989; 160: 938-943.

12. Chhabra BK. Nosocomial transmission of epidemic keratoconjunctivitis to food handlers in a nursing home. J Am Geriatr Soc 1995; 43: 1392-1393.

13. Tabery HM. Two outbreaks of adenovirus type 8 keratoconjunctivitis with different outcome. Acta Ophthalmol Scand 1995; 73: 358-360.

14. Colon LE. Keratoconjunctivitis due to adenovirus type 8: report on a large outbreak. Ann Ophthalmol 1991; 23: 63-65.

15. Threlkeld AB, Froggatt JW 3rd, Schein OD, Forman MS. Efficacy of a disinfectant wipe method for the removal of adenovirus 8 from tonometer tips. Ophthalmology 1993; 100: 1841-1845.

16. Cheung D, Bremner J, Chan JT. Epidemic kerato-conjunctivitis-do outbreaks have to be epidemic? Eye 2003; 17: 356-363.

17. Gordon YJ, Gordon RY, Romanowski E, Araullo-Cruz TP. Prolonged recovery of desiccated adenoviral serotypes 5 , 8 , and 19 from plastic and metal surfaces in vitro. Ophthalmology 1993; 100: 1835-1839.

18. Hutchinson AK, Coats DK, Langdale LM, Steed LL, Demmier $G$, Saunders RA. Disinfection of eyelid specula with clorhexidine gluconate (Hibiclens) after examinations for 
retinopathy of prematurity. Arch Ophthalmol 2000; 118: 786-789.

19. Rutala WA, Peacock JE, Grgen MF, Sobsey MD, Weber DJ. Efficacy of hospital germicides against adenovirus 8, a common cause of epidemic keratoconjunctivitis in health care facilities. Antimicrob Agents Chemother. 2006; 50: 1419-1424.
20. Trauzettel-Klosiniski S, Sundmacher R, Wigand R. The effects of topical steroids in epidemic kerato-conjunctivitis. Klin Monatsbl Augenheilkd 1980; 176: 899-906.

21. Laibson PR, Dhiri S, Oconer J, Ortolan G. Corneal infiltrates in epidemic keratoconjunctivitis. Response to double-blind corticosteroid therapy. Arch Ophthalmol 1970; 84: $36-40$. 